\title{
Lifetime of dynamic heterogeneity in strong and fragile kinetically constrained spin models
}

\author{
Sébastien Léonard and Ludovic Berthier ${ }^{1}$ \\ Laboratoire des Colloïdes, Verres et Nanomatériaux, UMR 5587 CNRS and Université \\ Montpellier II, 34095 Montpellier Cedex 5, France \\ E-mail: berthier@1cvn.univ-montp2.fr
}

Received 16 September 2005

Published 28 October 2005

Online at stacks.iop.org/JPhysCM/17/S3571

\begin{abstract}
Kinetically constrained spin models are schematic coarse-grained models for the glass transition which represent an efficient theoretical tool to study detailed spatio-temporal aspects of dynamic heterogeneity in supercooled liquids. Here, we study how spatially correlated dynamic domains evolve with time and compare our results to various experimental and numerical investigations. We find that strong and fragile models yield different results. In particular, the lifetime of dynamic heterogeneity remains constant and roughly equal to the alpha relaxation time in strong models, while it increases more rapidly in fragile models when the glass transition is approached.
\end{abstract}

\section{Introduction}

The viscosity of supercooled liquids increases extremely rapidly when the temperature is reduced towards the glass temperature. It is firmly established that this dramatic slowing down is spatially heterogeneous. Local relaxation is widely distributed in time-existence of broad stretched relaxations-but also in space-existence of dynamic heterogeneity [1]. The main physical aspect is that spatial fluctuations of local relaxations become increasingly spatially correlated when temperature decreases. Direct experimental investigations of the time and temperatures dependences of the relevant dynamic lengthscales at low temperature are however still missing.

To study dynamic heterogeneity, statistical correlators which probe more than two points in space and time have to be considered [1-3]. For example, if one wants to study spatial correlations of the local dynamics one has to define a two-point, two-time correlator,

$$
C_{2,2}(|i-j|, t)=\left\langle P_{i}(0, t) P_{j}(0, t)\right\rangle-\left\langle P_{i}(0, t)\right\rangle\left\langle P_{j}(0, t)\right\rangle,
$$

where notations are adapted to lattice spin models. In equation $(1),(i, j)$ denote lattice sites, $P_{i}(0, t)$ quantifies the dynamics at site $i$ between times 0 and $t$ (autocorrelation or persistence

1 Author to whom any correspondence should be addressed. 
functions), and brackets represent ensemble averages. The physical meaning of (1) is clear: given a spontaneous fluctuation of the two-time dynamics at site $i$, is there a similar fluctuation at site $j$ ? The quantity (1) has now been discussed both theoretically and numerically in some detail [3-5], generically revealing the existence of a growing spatial range of dynamic correlations in supercooled liquids accompanying an increasingly sluggish dynamics.

Logically, the next question is then: given spatial structures of the local relaxation between times zero and $t$, what will this structure look like, say, between times $t$ and $2 t$ ? In other words [6], how do dynamic heterogeneities evolve with time? This question is in fact simpler to address experimentally because no spatial resolution is needed and different experimental techniques can be devised: NMR [7], solvation dynamics [8], optical [9] and dielectric [10] hole-burning. In statistical terms, one wants to study a four-time correlation function of the general form

$$
C_{4}\left(t_{1}, t_{\mathrm{w}}, t_{2}\right)=\left\langle P_{i}\left(0, t_{1}\right) P_{i}\left(t_{1}+t_{\mathrm{w}}, t_{1}+t_{\mathrm{w}}+t_{2}\right)\right\rangle,
$$

which correlates dynamics between times zero and $t_{1}$ and between times $t_{1}+t_{\mathrm{w}}$ and $t_{1}+t_{\mathrm{w}}+t_{2}$. Again the physical content of (2) is clear [11]: given a dynamic fluctuation at site $i$ in a certain time interval $t_{1}$, how long does it take for this fluctuation to be washed out? This leads to the general concept of a lifetime, $\tau_{\mathrm{dh}}$, for dynamic heterogeneity. While many investigations $[1,2,7,8,10,12,13]$ indicate that $\tau_{\mathrm{dh}}$ is in fact slaved to the alpha-relaxation time of the liquid, $\tau_{\mathrm{dh}} \approx \tau_{\alpha}$, photobleaching experiments very close to the glass transition indicate that $\tau_{\mathrm{dh}}$ may become several orders of magnitude larger than $\tau_{\alpha}$, although in a surprisingly abrupt manner [14].

In this paper we study the lifetime of dynamic heterogeneity in kinetically constrained spin models of supercooled liquids [15]. These models represent schematic coarse-grained models for the glass transition and provide a very efficient tool to study in detail many spatio-temporal aspects related to dynamic heterogeneity such as dynamic lengthscales [16], scaling [17], or decoupling phenomena $[18,19]$. They are simple enough that analytical progress can be made and numerical simulations performed on a wide range of lengthscales and timescales, and yet rich enough that direct comparisons to both simulations and experiments can be made.

\section{Models}

Following previous works [16, 18-20], we focus on two specific spin facilitated models in one spatial dimension, namely the one-spin facilitated Fredrickson-Andersen (FA) model [21] and the East model [22], that respectively behave as strong and fragile systems [15]. These are probably the simplest models which incorporate the ideas that (i) mobility in supercooled liquids is both highly localized and sparse, as revealed by simulations [12]; (ii) a localized mobility very easily propagates to neighbouring regions: the dynamic facilitation concept. Detailed studies in spatial dimensions larger than one have shown that dimensionality does not play a relevant qualitative role $[17,18,23]$, and therefore justify the present one-dimensional studies.

Both models are defined by the same non-interacting Hamiltonian, $H=\sum_{i} n_{i}$, expressed in terms of a mobility variable, $n_{i}=1$ when site $i$ is mobile, $n_{i}=0$ otherwise. Dynamic facilitation is incorporated at the level of the dynamic rules through kinetic constraints. In the FA model, the site $i$ can evolve with Boltzmann probability if at least one of its two neighbours is mobile, $n_{i-1}+n_{i+1}>0$. In the East model the site $i$ can evolve only if its left neighbour is mobile, $n_{i-1}=1$.

We have performed numerical simulations of both models using a continuous time Monte Carlo algorithm where all moves are accepted and the time is updated according to the 

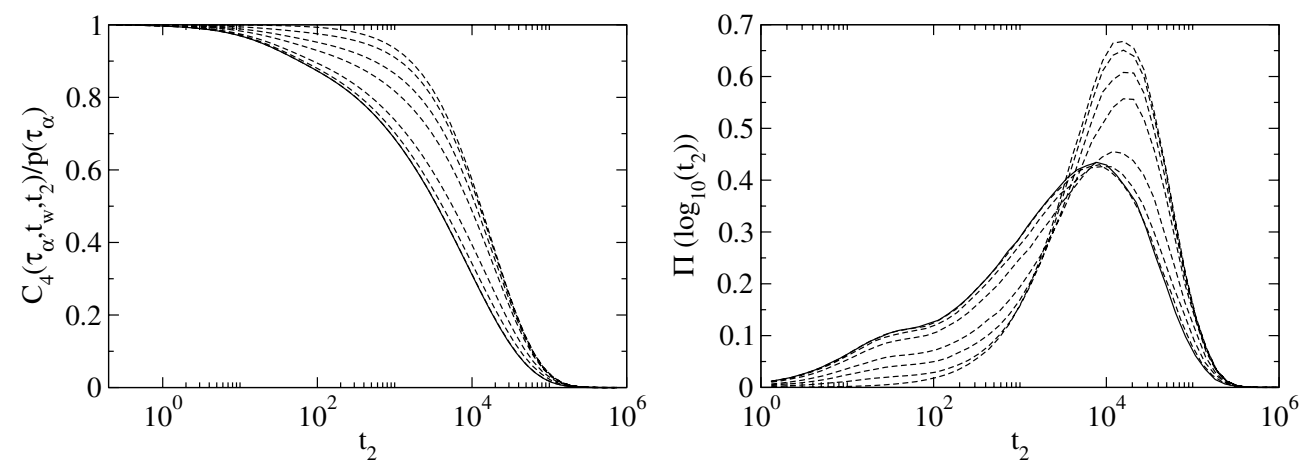

Figure 1. Left: four-time correlation function $C_{4}\left(\tau_{\alpha}, t_{\mathrm{w}}, t_{2}\right)$ normalized by its $t_{2}=0$ value for various $t_{\mathrm{W}}=0,550,2100,5200,20000,49500$, and 191000 (from right to left) in the FA model at $T=0.3$. These dashed curves converge at large $t_{\mathrm{w}}$ to the bulk persistence function, $p\left(t_{2}\right)$, shown as a full line. Right: corresponding logarithmic distributions of relaxation times.

corresponding statistical weight. Simulations have been performed 'only' over about seven decades in time because extensive time averaging is required to accurately measure multi-time correlation functions such as equation (2).

\section{Results}

\subsection{Dynamic filtering}

There are several parameters involved in the four-time correlator (2) that need to be appropriately chosen. Since dynamic heterogeneity is more pronounced for times close to $\tau_{\alpha}$ it is sensible to first fix $t_{1}=\tau_{\alpha}$ and to study the remaining $t_{\mathrm{w}}$ and $t_{2}$ dependences. As a local dynamic correlator we first focus on the persistence function [24], $P_{i}(0, t)=1$ if spin $i$ has not flipped in the interval $[0, t], P_{i}(0, t)=0$ otherwise. We also define the mean persistence, $p(t)=\left\langle P_{i}(t)\right\rangle$, from which we measure $\tau_{\alpha}$ via $p\left(\tau_{\alpha}\right)=e^{-1}$.

In figure 1 (left) we show the $t_{2}$ dependence of $C_{4}\left(\tau_{\alpha}, t_{\mathrm{w}}, t_{2}\right)$ for various $t_{\mathrm{w}}$ at $T=0.3 \mathrm{in}$ the FA model. We have normalized $C_{4}$ by $p\left(\tau_{\alpha}\right)=e^{-1}$, its value at $t_{2}=0$. By definition, this function describes the persistence function in the interval $\left[t_{\mathrm{w}}+\tau_{\alpha}, t_{\mathrm{w}}+\tau_{\alpha}+t_{2}\right]$ of those sites which had not flipped in the interval $\left[0, \tau_{\alpha}\right]$, and were therefore slower than average. The first term in the correlator (2) plays the role of a dynamic filter [11], selecting a sub-population of sites which have an average dynamics different from the bulk. From earlier works studying the spatial correlator (1), it is known that these sites belong to compact clusters that represent the largest regions of space with no mobility defects at time zero [16, 17, 23].

Immediately after filtering one expects therefore these slow regions to remain slow, as indeed observed in figure 1 for $t_{\mathrm{w}}=0$. When $t_{\mathrm{w}}$ increases, this selected population gradually forgets it was initially slow. When $t_{\mathrm{w}} \rightarrow \infty$, bulk dynamics is recovered,

$$
\frac{C_{4}\left(t_{1}, t_{\mathrm{w}} \rightarrow \infty, t_{2}\right)}{p\left(\tau_{\alpha}\right)} \rightarrow p\left(t_{2}\right),
$$

as demonstrated by the full line in figure 1. In figure 1 (right) we also show the (logarithmic) distribution of relaxation times corresponding to the functions shown in the left panel, a representation sometimes preferred in experimental works [1]. Both quantities are of course fully equivalent [20]. It is clear from these figures that once a subset of sites has been dynamically selected the remaining relaxation is narrower than the bulk relaxation. In fact, all 
persistence functions shown in figure 1 are well described by stretched exponentials. While $\beta=1 / 2$ is observed for the bulk dynamics, one finds $\beta \approx 0.83$ at $t_{\mathrm{w}}=0$. Accordingly, distributions of relaxation times progressively broaden when $t_{\mathrm{w}}$ increases. These results are consistent with experimental observations.

In the FA model their interpretation is straightforward. Stretching in this model follows from an exponential distribution of distance between mobility defects [16]. Dynamic filtering implies that this domain distribution is cut off at small distance. Narrower lengthscale distributions directly imply narrower timescale distributions.

We have also investigated the effect of changing the 'filter efficiency' [7], which in our case implies changing the duration of the filtering interval, $\left[0, t_{1}\right]$. While bulk distributions are found for $t_{1} / \tau_{\alpha} \ll 1$ (weak filtering), distributions shift to larger times and become very narrow when $t_{1} / \tau_{\alpha}$ increases. In the following we work at constant filtering, $t_{1}=\tau_{\alpha}$.

\section{2. 'Homogeneous' versus 'heterogeneous' dynamics}

The ability to select a sub-ensemble of sites that are slower than average is sometimes taken as a definition of dynamic heterogeneity [7], although lengthscales play no role in this view. That FA and East models display spatially heterogeneous dynamics is well known, and the results of the previous section are therefore natural.

Another indicator of dynamic heterogeneity has been proposed [11, 25, 26] based on the analysis of the four-time correlation (2). Consider the situation where $t_{\mathrm{W}}=0$, and $t_{1}=t_{2} \equiv t / 2$. In this case, one studies a 'three-time' correlation [26]

$$
F_{3}(t)=\left\langle P_{i}(0, t / 2) P_{i}(t / 2, t)\right\rangle
$$

Two extreme behaviours can be expected for $F_{3}(t)$. (i) Dynamics in the intervals $[0, t / 2]$ and $[t / 2, t]$ are totally uncorrelated, and thus $F_{3}(t) \approx[p(t / 2)]^{2}$. (ii) Dynamics in the two intervals are strongly correlated, in the sense that those regions that survive filtering in $[0, t / 2]$ are also those dominating the relaxation in the full interval $[0, t]$. In that case, $F_{3}(t) \approx p(t)$. Scenarios (i) and (ii) have been termed 'homogeneous' and 'heterogeneous', respectively, although again lengthscales play no role in the distinction. Clearly, both estimates become equivalent when $p(t)$ decays exponentially.

Of course when studying the persistence function in the FA and East models, scenario (ii) strictly applies by definition, because $P_{i}(0, t / 2) P_{i}(t / 2, t)=P_{i}(0, t)$. In real materials, smoother dynamic functions are studied, directly defined from the particle positions instead of a mobility field. Our strategy is therefore to couple probe particles to our mobility field; see $[18,19]$ for technical details. From probe molecule displacements, $\delta x(0, t)=x(t)-x(0)$, we define self-intermediate scattering functions, $F_{s}(k, t)=\langle\cos [k \cdot \delta x(0, t)]\rangle$, and the analogue of equation (4), $F_{3}(k, t)=\langle\cos [k \cdot \delta x(0, t / 2)] \cos [k \cdot \delta x(t / 2, t)]\rangle$.

Our numerical results are presented in figure 2. Clearly, the time dependence of $F_{3}$ closely follows the one of $F_{s}(k, t)$, in agreement with the 'heterogeneous' scenario described above. This is consistent with numerical results [26].

In the present approach, this result is a natural consequence of decoupling between structural relaxation and diffusion $[18,19]$. At large wavevectors, $k \sim \pi$, corresponding to distances of the order of the lattice spacing, $F_{s}(k, t)$ is dominated by the time distribution of the first jump of the probe molecule in the interval $[0, t]$, so that $F_{s}(k, t) \approx p(t) \approx F_{3}(k, t)$. At large distances, $k<k^{*}$, Fickian diffusion holds [19], $F_{s}(k, t)=\exp \left(-k^{2} D_{s} t\right)$, and there is no distinction between homogeneous and heterogeneous relaxation. At intermediate wavevectors, $\pi>k>k^{*}$, the long-time decay of $F_{s}(k, t)$ is again dominated by the persistence time distribution, because the timescale it takes a molecule to make $2 \pi / k$ steps is strongly 

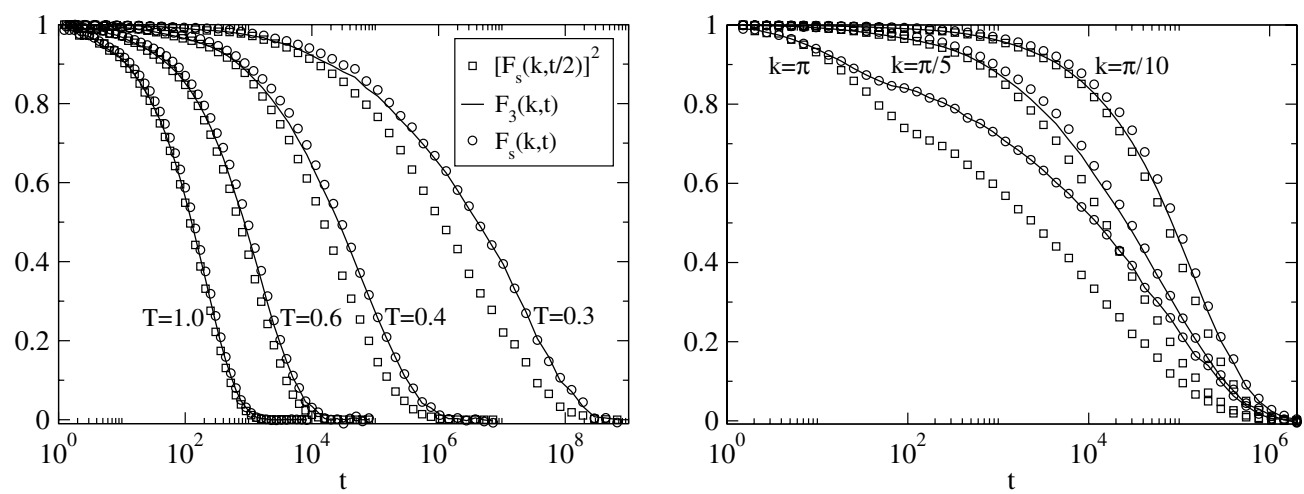

Figure 2. $F_{3}(k, t)$ and its 'heterogeneous', $F_{s}(k, t)$, and 'homogeneous', $\left[F_{s}(k, t / 2)\right]^{2}$, limits. Left: East model for fixed $k=\pi / 5$ and various temperatures. Right: East model at $T=0.4$ and various wavevectors.

dominated by the timescale to make the first step [19]. This is just the condition for the heterogeneous scenario to hold, in agreement with figure 2. The characteristic wavevector separating the two regimes, $k^{*}(T)=1 / \sqrt{\tau_{\alpha} D_{s}}$, decreases when temperature decreases, opening a larger heterogeneous window; $k^{*}$ also sets the upper limit of validity of Fickian diffusion in supercooled liquids $[19,27]$.

\subsection{Lifetime of dynamic heterogeneity}

After dynamic filtering it takes some time for filtered distributions to re-equilibrate towards the bulk relaxation, cf figure 1. To extract the typical lifetime of dynamic heterogeneity, $\tau_{\mathrm{dh}}$, we tried several procedures, which all lead to similar results, based on how timescales (time decay of persistence functions or moments of the corresponding distributions) return to their equilibrium values. Following $[13,18]$ we also measured the integrated difference between filtered and bulk dynamics, $\Delta\left(t_{\mathrm{w}}\right) \equiv \int_{0}^{\infty} \mathrm{d} t_{2}\left[C_{4}\left(\tau_{\alpha}, t_{\mathrm{w}}, t_{2}\right) / p\left(\tau_{\alpha}\right)-p\left(t_{2}\right)\right]$. From figure 1 , we expect that $\Delta\left(t_{\mathrm{W}}\right)$ goes to zero on a timescale $\tau_{\mathrm{dh}}$. In practice, we define $\tau_{\mathrm{dh}}$ as $\Delta\left(\tau_{\mathrm{dh}}\right) / \Delta(0)=e^{-1}$. In principle, $\tau_{\mathrm{dh}}$ depends on the filtering time, $t_{1}$, and on temperature, $T$.

We show in figure 3 results at various $T$ but constant filter efficiency, $t_{1}=\tau_{\alpha}(T)$, in East and FA models. While $\tau_{\mathrm{dh}}$ is set by $\tau_{\alpha}$ in the FA model (the tiny deviation observed in figure 3 is due to finite $T$ corrections, which weaken when $T$ gets lower), this is not true in the East model, where $\tau_{\mathrm{dh}}$ systematically grows faster than $\tau_{\alpha}$ at low $T$, as emphasized in the inset. Quantitatively a power law relationship, $\tau_{\mathrm{dh}} \sim \tau_{\alpha}^{1+\zeta}$, with $\zeta \approx 0.06$, is a good description of the data, although an alternative fitting formula could probably be used.

In the fragile case, $\tau_{\mathrm{dh}}$ can therefore be considered as an additional slow timescale characterizing the alpha relaxation [14], on top of $\tau_{\alpha}$ and $1 / D_{s}$ [18]. The comparative study of FA and East models offers a possible physical interpretation. While both models display stretched relaxations, in the FA model stretching is constant, $\beta=1 / 2$, while $\beta$ increases linearly with $T$ in the East model [20]. Therefore, $\tau_{\alpha}$ represents the first moment of a distribution that becomes wider and wider when $T$ decreases. We attribute the small but systematic decoupling between $\tau_{\mathrm{dh}}$ and $\tau_{\alpha}$ to this broadening.

However, this decoupling does not explain the results of photobleaching experiments, which show that $\tau_{\mathrm{dh}} / \tau_{\alpha}$ increases strongly close to $T_{g}$ [14]. In OTP, while $\tau_{\alpha}$ changes by about one decade from $T_{g}+4 \mathrm{~K}$ to $T_{g}+1 \mathrm{~K}, \tau_{\mathrm{dh}} / \tau_{\alpha}$ changes by two orders of magnitude, so that 

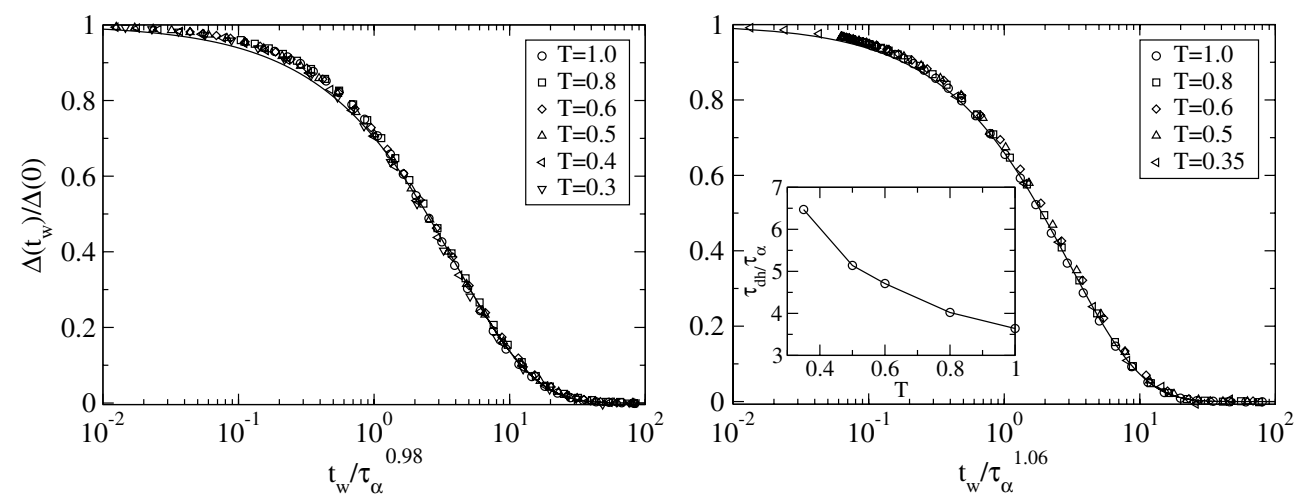

Figure 3. Integrated difference between filtered and bulk dynamics at constant filtering time, $t_{1}=\tau_{\alpha}$, and various temperatures in the FA (left) and East (right) models. Times have been rescaled to collapse the data points at various temperatures and extract the lifetime of dynamic heterogeneity, $\tau_{\mathrm{dh}}$. In the FA model, $\tau_{\mathrm{dh}}$ is set by $\tau_{\alpha}$ with tiny corrections that vanish at low $T$. In the East model, $\tau_{\mathrm{dh}}$ grows faster than $\tau_{\alpha}$, as emphasized in the inset showing the systematic increase of $\tau_{\mathrm{dh}} / \tau_{\alpha}$ as $T$ decreases. Master curves are fitted by stretched exponentials shown with full lines, $\beta=0.75$ (left) and $\beta=0.8$ (right).

$\zeta \approx 2$. This value is much too large to be accounted for by the above results. Presumably, also, $\beta$ does not vary much on such a tiny temperature interval. From the present perspective, additional phenomena such as non-equilibrium effects due to the proximity of $T_{g}$ must be invoked to understand photobleaching experiments. Instead, we were able to predict a smaller, but definitely non-vanishing, decoupling between the lifetime of dynamic heterogeneity and the alpha-relaxation time which could be detected in dynamic filtering experiments performed in a sufficiently large temperature window in fragile glass-formers.

\section{References}

[1] Ediger M D 2000 Annu. Rev. Phys. Chem. 5199

[2] Sillescu H 1999 J. Non-Cryst. Solids 24381

[3] Glotzer S C 2000 J. Non-Cryst. Solids 274342

[4] Bennemann C, Donati C, Baschnagel J and Glotzer S C 1999 Nature 399246

Lacevic N, Starr F W, Schrøder T B and Glotzer S C 2003 J. Chem. Phys. 1997372

[5] Toninelli C, Wyart M, Berthier L, Biroli G and Bouchaud J-P 2005 Phys. Rev. E 71041505

[6] Doliwa B and Heuer A 2002 J. Non-Cryst. Solids 307-310 32

[7] Schmidt-Rohr K and Spiess H W 1992 Phys. Rev. Lett. 663020

Böhmer R, Diezemann G, Hinze G and Sillescu H 1998 J. Chem. Phys. 108890

[8] Yang M and Richert R 2001 J. Chem. Phys. 1152676

[9] Ediger M D 1995 J. Chem. Phys. 103752

[10] Schiener B, Böhmer R, Loidl A and Chamberlin R V 1996 Science 274752

[11] Heuer A 1997 Phys. Rev. E 56730

[12] Perera D N and Harrowell P 1999 J. Chem. Phys. 1115441

[13] Flenner E and Szamel G 2004 Phys. Rev. E 70052501

[14] Wang C-Y and Ediger M D 1999 J. Phys. Chem. B 1034177

[15] Ritort F and Sollich P 2003 Adv. Phys. 52219

[16] Garrahan J P and Chandler D 2002 Phys. Rev. Lett. 89035704

[17] Whitelam S, Berthier L and Garrahan J P 2004 Phys. Rev. Lett. 92185705

Whitelam S, Berthier L and Garrahan J P 2005 Phys. Rev. E 71026128

Berthier L 2003 Phys. Rev. Lett. 91055701

[18] Jung Y, Garrahan J P and Chandler D 2004 Phys. Rev. 69061205 
Jung Y, Garrahan J P and Chandler D 2005 J. Chem. Phys. 123084509

[19] Berthier L, Chandler D and Garrahan J P 2005 Europhys. Lett. 69230

[20] Berthier L and Garrahan J P 2003 J. Chem. Phys. 1194367

Berthier L and Garrahan J P 2003 Phys. Rev. E 68041201

[21] Fredrickon G H and Andersen H C 1984 Phys. Rev. 531244

[22] Jäckle J and Eisinger S 1991 Z. Phys. B 84115

[23] Berthier L and Garrahan J P 2005 J. Phys. Chem. B 1093578

[24] Heuer A, Tracht U and Spiess H W 1997 J. Chem. Phys. 1073813

[25] Heuer A and Okun K 1997 J. Chem. Phys. 1066176

[26] Doliwa B and Heuer A 1998 Phys. Rev. Lett. 804915

[27] Berthier L 2004 Phys. Rev. E 69 020201(R) 